\title{
Autophagic and Apoptotic Effects of HDAC Inhibitors on Cancer Cells
}

\author{
Hidemi Rikiishi \\ Department of Microbiology and Immunology, Tohoku University Graduate School of Dentistry, 4-1 Seiryo-machi, \\ Aoba-ku, Sendai 980-8575, Japan \\ Correspondence should be addressed to Hidemi Rikiishi, riki@m.tains.tohoku.ac.jp
}

Received 23 June 2010; Accepted 3 March 2011

Academic Editor: Patrick Matthias

Copyright (C) 2011 Hidemi Rikiishi. This is an open access article distributed under the Creative Commons Attribution License, which permits unrestricted use, distribution, and reproduction in any medium, provided the original work is properly cited.

Because epigenetic alterations are believed to be involved in the repression of tumor suppressor genes and the promotion of tumorigenesis in cancers, novel compounds endowed with histone deacetylase (HDAC) inhibitory activity are an attractive therapeutic approach. Indeed, the potential of HDAC inhibitors for cancer therapy has been explored in preclinical models, and some agents approved for hematologic malignancies have reached the clinical setting. HDAC inhibitors are able to mediate the induction of both apoptosis and autophagy, which are related to anticancer activity in a variety of cancer cell lines. Given the inherent resistance to apoptosis that characterizes cancer, the targeting of alternative pathways is an attractive strategy to improve anti-tumor therapy. The activation of autophagy represents novel cancer treatment targets. This paper aims to critically discuss how the anticancer potential of HDAC inhibitors may elicit a response to human cancers through different cell pathways leading to cell death.

\section{Introduction}

An ideal anticancer agent should be toxic to malignant cells with minimum toxicity towards normal cells. Currently, there are only a limited number of such agents available for clinical use; thus, the development of novel cancer-selective drugs is an important and challenging task. Caspasemediated apoptosis, involving both intrinsic and extrinsic pathways, is the main mechanism of action of most current anticancer treatments. Several anticancer drugs have been shown to induce autophagy as well as apoptosis. Autophagy is a catabolic pathway whereby cytoplasmic proteins and organelles are sequestered in vacuoles and delivered to lysosomes for degradation and recycling; however, the role of autophagy in cancer development and in the response to therapy is still controversial, requiring further investigation.

Chemoresistance may be due to epigenetic alterations leading to defects in the apoptotic pathway; therefore, modulating epigenetic changes may increase the efficacy of chemotherapy. It is possible to interfere with epigenetic modifications, such as histone acetylation, using relatively specific and reversible inhibitors. In addition, the induction of autophagy has been also observed in malignant cells following treatment with histone deacetylase (HDAC) inhibitors [1]. HDAC inhibitors are promising new agents for the treatment of multiple myeloma as they promote hyperacetylation and activate chromatin remodeling. HDAC inhibition leads to many changes at the molecular and cellular levels. HDAC inhibitors preferentially kill transformed cells or cancer cells in both cell cultures and animal models. Although further investigation is required to fully elucidate the consequences of both autophagy and apoptosis induction by treatment with HDAC inhibitors, the pharmacological inhibition of HDAC provides a new therapeutic basis for targeting cell death processes.

\section{HDAC Family}

In addition to DNA mutations (genetic changes), most cells in multicellular organisms result from aberrations in chromatin-modifying proteins, such as HDAC and DNA methyltransferase (epigenetic changes). It became 
increasingly evident that cancer formation and persistence may be caused not only by genetic mutations but also by changes in the patterns of epigenetic modifications [2]. Epigenetic modifications are of profound importance to cells and appear to play roles in many, if not all, biological processes throughout life. In particular, acetylation and deacetylation of the lysine residues on histone proteins play a key role in the regulation of gene transcription [3]. Whereas histone acetyltransferase catalyzes the acetylation of histones and relaxes chromatin to increase the accessibility of transcription factors to the promoters of the target genes, HDACs remove the acetyl groups from histones and repress transcription [4]. In the discovery of drugs, HDAC has become a novel target for the treatment of cancer and other diseases. Inhibition of HDAC activity leads to the acetylation of histones and nonhistone proteins, and alterations in target gene expression are related to changes induced by HDAC inhibition.

According to phylogenetic analyses and sequence homology, the HDAC family consists of at least 18 members divided into two families and four classes in eukaryotic cells. These two families are the classical and sirtuin families. HDACs are categorized into class I (HDACs 1, 2, 3, and 8), class IIa (HDACs 4, 5, 7, and 9), class IIb (HDACs 6 and 10), class III (SIRTs), and class IV (HDAC11) [5]. Class I and II have zinc as a cofactor, so they are hydrolases which contain zinc. Class III, which is a series of NAD-dependent Sir2 families, differs from other HDAC classes. Class I and II HDACs are mainly found to be involved in cancer pathogenesis. Class I are found exclusively in the nucleus, whereas in certain cellular signals, class II shuttle between the nucleus and cytoplasm. While human HDACs are well characterized in terms of their catalytic domains, the discovery of type-specific inhibitors is important for anticancer therapies.

\section{HDAC Inhibitors}

HDAC inhibitors are emerging as potent anticancer agents that can reactivate gene expression and restore the capability of malignant cells to undergo programmed cell death. Several natural and synthetic compounds are currently known to inhibit HDACs (Table 1). Since HDAC inhibitors do not inhibit all HDAC isoforms to the same extent, these agents can be grouped into pan- and class I-specific inhibitors [5]. Hydroxamic acids (TSA, SAHA, LAQ824, and CBHA) and pyroxamic acids (PXD101 and CRA-026440) are panHDAC inhibitors targeting class I, II, and IV HDACs in the nanomolar range [17]. In contrast, carboxylic acids (valproic acid and sodium butyrate) and benzamides (MS275, CI994, and MGCD0103) or cyclic tetrapeptides (trapoxin, depsipeptide and spiruchostatin A) are rather class I-specific HDAC inhibitors [18].

HDACs are often overexpressed in various types of cancers, compared with the corresponding normal tissues, and their overexpression is correlated with a poor prognosis [19]. Currently, at least 15 different HDAC inhibitors are being used in clinical trials as a part of mono- or combination therapies. For example, the orally available pan-inhibitor LBH589 (panobinostat) is currently being developed for several hematological malignancies. SAHA (vorinostat) has been approved for marketing based on data from a large Phase II trial of patients with cutaneous Tcell lymphoma that demonstrated a $30 \%$ objective response rate [6]. The class I-selective intravenously delivered agent FK228 (romidepsin), has also shown promising efficacy in patients with cutaneous T-cell lymphoma, with an overall objective response rate of $32 \%$. The class I-selective orally available compound, MGCD0103 (mocetinostat), is in development for multiple solid and hematologic cancers [13]. Clinical trials of several HDAC inhibitors include patients with a wide variety of hematologic and solid neoplasms, including chronic lymphatic leukemia, Hodgkin's lymphoma, myeloproliferative disorder, B-cell lymphoma, acute myeloid leukemia, multiple myeloma, head and neck cancer, brain tumors, melanoma, lung cancer, hepatocellular cancer, breast cancer, ovarian cancer, renal cell carcinoma, and pancreatic adenocarcinoma. However, the main side effects of HDAC inhibitor, SAHA, are fatigue, nausea, anorexia, diarrhea, thrombus formation, thrombocytopenia, neutropenia, anemia, myalgia, hypokalemia and hypophosphatemia. Reversible thrombocytopenia has been seen with almost all HDAC inhibitors in the clinic and appears to be a mechanism-related class effect. By targeting only the most relevant HDAC isoform, it may be possible to greatly improve the efficacy by removing certain toxicities that may be associated with the inhibition of multiple isoforms.

\section{Programmed Cell Death (PCD)}

Cell death is a result of an unsuccessful cytoprotective mechanism against intracellular and extracellular stressors, and it is broadly divided into three forms: apoptosis, autophagy, and necrosis (Table 2). Morphologically, type I cell death (apoptosis) is characterized by chromatin condensation and DNA fragmentation. Biochemically, apoptosis is characterized by double-stranded cleavage at the linker regions between nucleosomes, resulting in the formation of multiple DNA fragments, and phosphatidylserine externalization accompanied by a series of gene and protein expressions. In the extrinsic pathway, apoptosis is mediated by death receptors on the cell surface, while in the intrinsic pathway, mitochondria play an important role. In both pathways, activated caspases (cysteine aspartic acid-specific proteases) cleave their substrates and activate other downstream cellular substrates. Type II cell death (autophagy) is characterized by the massive accumulation of double-membrane vesicles, commonly referred to as autophagosomes. In general, autophagy promotes the survival of stress; however, there is increasing evidence that when autophagy is overstimulated, it can progress to autophagic cell death. It has been also documented that malignant cell types undergo autophagic cell death when responding to anticancer agents, indicating the potential utility of autophagic cell death induction in cancer therapy. Type III cell death (necrosis) is characterized by oncosis and plasma membrane rupture [20]. Necrosis is an uncontrolled cell death manifesting osmotic dispersion of cells and organelles. While apoptosis involves the rapid destruction of all cellular structures and organelles, 
TABLE 1: Characteristics of HDAC inhibitors in clinical trials.

\begin{tabular}{|c|c|c|c|c|}
\hline Chemistry & Compounds & HDAC Targets & Clinical trials & Ref \\
\hline \multirow{6}{*}{ Hydroxymates } & SAHA (vorinostat) & Classes I, II, and IV & Phase III* & {$[6]$} \\
\hline & PXD101 (belinostat) & Classes I, and IIa, HDAC6 & Phase II & {$[7]$} \\
\hline & Trichostatin A & Classes I and II & Toxic & {$[8]$} \\
\hline & LAQ824 (dacinostat) & Classes I and II & Phase I & {$[9]$} \\
\hline & PCI24781 & Classes I and IIb & Phase I & [10] \\
\hline & LBH589 (panobinostat) & Classes I and IIa & Phase II & [11] \\
\hline Cyclic tetrapeptides & FK228 (romidepsin) & HDAC1, 2, 4, 6 & Phase II & {$[12]$} \\
\hline \multirow{2}{*}{ Benzamides } & MGCD0103 (mocetinostat) & HDAC1, 2, 3, 11 & Phase II & {$[13]$} \\
\hline & MS275 (entinostat) & HDAC1, 2, 3, 9 & Phase II & [14] \\
\hline \multirow{2}{*}{ Short-chain fatty acids } & Valproic acid & Classes I and IIa & Phase II & {$[15]$} \\
\hline & Butyrate & Classes I and IIa & Phase II & [16] \\
\hline
\end{tabular}

* Approved (cutaneous T-cell lymphoma).

autophagy is a slow, spatially restricted phenomenon in which parts of the cytoplasm are sequestered within doublemembraned vacuoles and finally digested by lysosomal hydrolases [21].

\section{Apoptosis Induced by HDAC Inhibitors}

All HDAC inhibitors have been reported to activate either an extrinsic or intrinsic pathway or both of these cell death pathways in many cancer models [22]. The death-receptor pathway is activated when ligands, such as Fas or TRAIL, bind to their death receptors. It was observed that MS275 (entinostat) as well as SAHA induced TRAIL expression without altering DR4 or DR5 levels in breast cancer cells, which was mediated via SP1 and markedly enhanced adriamycin cytotoxicity in these cells [23]. This resulted in the recruitment of an adaptor protein, FADD, and the activation of caspase-8. The mitochondrial pathway is activated by stress stimuli (chemotherapeutic agents) that disrupt the mitochondrial membrane, causing the release of proteins, including cytochrome $c$ and SMAC. Cytochrome $c$ release leads to apoptosome formation and activation of caspase-9. Caspase- 8 and caspase- 9 can then cleave caspases$3,-6$, and -7 , culminating in apoptosis. SAHA and MS275 induced mitochondrial dysfunction and apoptosis through enhanced ROS generation, XIAP downregulation and JNK1 activation [24]. Various pro- (Bax, Bak, Bim, Bid, etc.) and antiapoptotic (Bcl-2, Bcl-xL, Mcl-1, etc.) proteins regulate these cell death pathways. HDAC inhibitors, including TSA, FK228, SAHA, and LBH589, decrease the expression of $\mathrm{Bcl}-2, \mathrm{Bcl}-\mathrm{xL}$ and XIAP, and enhance the expression of proapoptotic proteins, such as Bax and Bak, thereby enhancing TRAIL-mediated cytotoxicity in a variety of cancer cells via the amplification of intrinsic as well as extrinsic apoptotic pathways [25]. Using siRNA approaches targeting Bim, the contribution of Bim to TSA-induced mitochondrial apoptosis was confirmed [26]. In addition, serine proteasedependent and caspase-independent apoptosis is induced by treatment with the pan-HDAC inhibitor, TSA, in pancreatic ductal adenocarcinoma cell lines [27]. This process correlates with the initially increased expression of the multidomain proapoptotic Bcl-2 family member, Bax, and the subsequent release of AIF and Omi/Htr-A2 from mitochondria. The Bax tumor suppressor gene is downregulated by SIRT1 through the DNA end-binding factor, Ku70. In the absence of SIRT1, Ku70 might be hyper-acetylated, leading to the disruption of Ku70-Bax interaction. This in turn releases more Bax, which promotes apoptosis [28]. HDAC2 inhibition opens the locus of the epigenetically silenced NOXA gene, a BH3-only protein and apical initiator of apoptosis [29]. Given the multitude of cellular effects triggered by HDAC inhibitors, it is probable that several different mechanisms contribute to their anticancer activity. Another mechanism of cancer cell death resulting from HDAC inhibitor treatment is discussed below.

\section{Autophagy Induced by HDAC Inhibitors}

While autophagy has become one of the most attractive topics in cancer research, the current autophagy studies are often viewed as confusing, because of its association with apparently contradictory roles, such as survival and cell death, depending on the model used [30]. A number of anticancer therapies, including HDAC inhibitors, have been observed to induce autophagy in human cancer cell lines.

6.1. Prosurvival Functions. Autophagy is an evolutionarily conserved cell survival pathway that enables cells to recoup ATP and other critical biosynthetic molecules during nutrient deprivation or exposure to hypoxia, which are hallmarks of the tumor microenvironment. The disruption of autophagy with the antimalarial drug, chloroquine, strongly enhanced SAHA-mediated apoptosis in colon cancer cells, knockdown of the essential Atg7 also sensitized cells to SAHA-induced apoptosis [31]. HDAC6, a class II HDAC isotype, is directly involved in the autophagic degradation of the cell; therefore, drugs that impair autophagy could theoretically potentiate the anticancer efficacy of SAHA and other HDAC inhibitors by disabling this important cell survival mechanism. 
6.2. Prodeath Functions. The cytotoxic effects of autophagy may be explained by the extensive autophagic degradation of intracellular content or by the interfacing of the autophagic process to proapoptotic signals. Persistent autophagy in response to cellular stress states serves as a potent death signal, as in the case of therapy-induced autophagy, a specific nonapoptotic death pathway triggered upon exposure to chemotherapeutic compounds. In general, autophagic cell death is caspase independent and does not involve classic DNA laddering; however, the mechanisms underlying autophagic cell death are mostly undefined. A link between autophagy and related autophagic cell death has been demonstrated using pharmacological (3-methyladenine) and genetic (silencing of Atg5, Atg7, and Beclin-1) approaches for the suppression of autophagy. In chondrosarcoma cell lines, SAHA induced autophagy-associated cell death, as shown by the detection of autophagosome-specific protein and specific ultrastructural morphology in the cytoplasm [32]. In HeLa cells, HDAC inhibitors (butyrate and SAHA) can induce both mitochondria-mediated apoptosis and caspaseindependent autophagic cell death. HeLa cells with APAF-1 knockout or Bcl-xL overexpression were induced to undergo autophagic cell death when cultured with SAHA or butyrate [1]. Therefore, it is reasonable to propose that the induction of autophagic cell death may be used as a therapeutic strategy to treat cancer.

\section{Molecular Mechanisms of HDAC-Induced Autophagy}

Many signaling pathways, including mTOR, AIF, ROS, CDKs, and HDAC1/6, play important roles in regulating HDAC-induced autophagy (Table 3). Their molecular mechanisms and regulation of autophagy are discussed below.

7.1. mTOR. mTOR plays a role in the initiation and maturation of autophagy by controlling signal transduction cascades involved in this process, acts as a good gatekeeper in autophagy, and exerts an inhibitory effect on autophagy [40]. Both mTOR and phospho-mTOR expressions were strongly decreased in SAHA-treated endometrial stromal sarcoma (ESS-1) cells in a concentration-dependent manner [33]. It seems that SAHA modulates the stability and/or the degradation mechanisms of mTOR at the protein level. SAHA also exerted an influence on mTOR phosphorylation. The level of phospho-S6rp, which plays a regulatory role in the mTOR pathway, was also affected by SAHA. S6rp phosphorylation is directly involved in the control of autophagic processes. The same results were found in ESS-1 treated by rapamycin, a well-known mTOR inhibitor. Further investigations are needed to elucidate the exact pharmacological mechanisms of different HDAC inhibitors in different experimental systems and to clarify whether HDAC inhibitors modulate the expression and phosphorylation of mTOR directly or act somewhere upstream of mTOR.

7.2. AIF. It was examined in malignant rhabdoid tumor cells whether HDAC-inhibitor- (FK228-) mediated autophagy involved AIF, which acts as a safeguard executioner in cancer cells with faulty caspase activation [34]. FK228 treatment induced the redistribution of AIF to the nucleus, in either the presence or absence of zVAD-fmk. siRNA against AIF prevented the redistribution of LC3 into punctate cytoplasmic structures and blocked FK228-induced autophagy. AIF siRNA also decreased the conversion of LC3I to LC3-II, as analyzed by Western blotting. Treatment with autophagy inhibitors (chloroquine or 3-methyladenine) increased FK228-induced cell death. These findings suggest that AIF contributes to the autophagy induced by FK228, and disrupting autophagy enhances FK228-induced apoptosis.

7.3. ROS. Targeting the autophagy pathway might enhance the anticancer activity of SAHA, and autophagy inhibitors would synergistically potentiate the proapoptotic effects of SAHA. SAHA has pleiotropic effects that contribute to its mechanism of action in malignant cells, including the generation of ROS, and this has been shown to be a critical event in SAHA-induced cell death [41]. To determine whether chloroquine may modulate SAHA-induced ROS generation, the intracellular levels of the oxygen radical superoxide $\left(\mathrm{O}_{2}{ }^{-}\right)$were quantified after $12 \mathrm{~h}$ treatment with chloroquine, SAHA, and the combination of chloroquine and SAHA in K562 and LAMA 84 cells [35]. In both cell lines, there were marked increases in the generation of $\mathrm{O}_{2}{ }^{-}$ in cells treated with a combination of chloroquine and SAHA compared with cells treated with either single agent. Cells pretreated with $\mathrm{N}$-acetyl-cysteine displayed significantly reduced apoptosis induction in response to chloroquine and SAHA. It is possible that the initial ROS release triggered autophagy as a protective defense mechanism of cancer cells to deal with SAHA, but the temporal order of protective autophagy is followed by apoptosis, as if autophagy sets the stage for apoptosis to kill prostate cancer cells after a futile attempt to rescue them from death.

7.4. $p 21^{\mathrm{CIP} / \mathrm{WAF} 1}$. Both a novel sulfur-containing hydroxamate HDAC inhibitor, H40, and SAHA induced autophagy in prostate cancer $\mathrm{PC}-3 \mathrm{M}$ cells in a concentrationdependent manner, which correlated well with their cytotoxic effects. These HDAC inhibitors significantly increased CDK inhibitor $\mathrm{p} 21^{\mathrm{CIP} / \mathrm{WAF} 1} \mathrm{mRNA}$ and protein expression in both PC-3M and HL-60 cells in concentrations within their cytotoxic ranges [36]. The upregulation of p21 CIP/WAF1 correlated with reduced sensitivity and blocked the apoptotic mechanism, and these cells seemed to die by autophagy. Recently, silencing of HDAC3 has been found to induce the expression of $\mathrm{p} 21^{\mathrm{CIP} / \mathrm{WAF} 1}$ and cell cycle arrest in the G2/M phase in colon cancer cells [42]. HDAC4 was shown to suppress $\mathrm{p} 21^{\mathrm{CIP} / \mathrm{WAF} 1}$ in ovarian carcinoma cells, cervical cancer cells, glioblastoma cells and breast cancer cells in a nonredundant fashion [43]. These results suggest that the presence of $\mathrm{p} 21^{\mathrm{CIP} / \mathrm{WAF} 1}$ determines which type of PCD the cell undergoes.

7.5. HDAC1 and HDAC6. HDAC inhibition leads to many changes at the molecular and cellular levels. Class I HDAC 
TABLE 2: Characteristic features of programmed cell death.

\begin{tabular}{|c|c|c|c|}
\hline Programmed cell death (PCD) & Feature & Key regulators & Measurements \\
\hline \multirow{3}{*}{ Apoptosis (type I PCD) } & Chromatin condensation & Caspases & Caspase activation \\
\hline & DNA laddering & Cytochrome $c$ & DNA fragmentation \\
\hline & $\begin{array}{l}\text { Blebbing (nuclear, cytoplasmic) } \\
\text { Apoptotic bodies }\end{array}$ & Bcl-2 family members & Annexin V \\
\hline \multirow{3}{*}{ Autophagy (type II PCD) } & Blebbing & \multirow{3}{*}{$\begin{array}{l}\text { Autophagy. genes: beclin, LC3, Atg1, } \\
\text { Atg5, and Atg7 }\end{array}$} & LC3 localization \\
\hline & Autophagic vesicles & & \\
\hline & $\begin{array}{l}\text { Degradation of Golgi } \\
\text { No DNA laddering }\end{array}$ & & Autophagic vesicles \\
\hline \multirow{3}{*}{ Necrosis (type III PCD) } & Swollen organelles & \multirow{3}{*}{ RIPK1, TRAF2, PARP, and Calpains } & Extracellular HMGB \\
\hline & Random DNA degradation & & \\
\hline & $\begin{array}{l}\text { Cytoplasmic membrane rupture } \\
\text { Potent inflammatory response }\end{array}$ & & S100 family members \\
\hline
\end{tabular}

TABLE 3: Effects of HDAC inhibitor on the process of autophagy.

\begin{tabular}{lcccr}
\hline Mechanisms & HDAC inhibitors & Cell lines* & Detection methods & Ref \\
\hline mTOR & SAHA & ESS-1 & Phosphorylation (mTOR) & {$[33]$} \\
AIF & FK228 & MRT & siRNA (AIF) & {$[34]$} \\
ROS & SAHA & K562, LAMA84 & N-acetyl-cysteine, chloroquine & {$[35]$} \\
p21 CIP/WAF1 & H40, SAHA & PC-3M, HL-60 & mRNA, protein expression & {$[36]$} \\
HDAC1 & FK228 & HeLa & siRNA (HDAC1) & {$[37]$} \\
HDAC6 & Tubacin & MEF & siRNA (HDAC6) & {$[38]$} \\
Mitochondria & LBH589, LAQ824 & E $\mu$ - $m y$ c lymphoma & Knockout (Apaf-1, caspase-9) & {$[39]$} \\
\hline
\end{tabular}

*ESS-1: endometrial stromal sarcoma cells; LAMA84: human chronic myeloid leukemia; MEF: mouse embryonic fibroblasts; MRT: malignant rhabdoid tumors.

inhibitor, FK228, led to autophagic activities through the formation of autophagic vacuoles and the increase of acidic compartments in HeLa cells. When HDAC1 siRNA was used, the amount of HDAC1 mRNA was significantly decreased in knocked-down cells, and HDAC1 inhibition induced the conversion of LC3-I to LC3-II which leads to autophagy in HeLa cells [37]. Thus, HDAC1 may play a role in autophagy because the inhibition of HDAC1 with a specific inhibitor or siRNA can induce autophagy.

HDAC6 is a member of the class II HDAC family and is known to deacetylate $\alpha$-tubulin and increase cell motility. The ubiquitin-binding deacetylase, HDAC6, was also identified as a central component of basal autophagy that targets protein aggregates and damages mitochondria; however, HDAC6 is not required for autophagy activation; rather, it controls the fusion of autophagosomes to lysosomes [38]. HDAC6 promotes these fusion events by recruiting a cortactin-dependent, actin-remodelling machinery to ubiquitinated protein aggregates, where the assembly of F-actin facilitates autophagosome-lysosome fusion and clearance of autophagic substrates. In addition, the observation that tubacin, a selective inhibitor of HDAC6 deacetylase activity, blocks LC3 recruitment suggests that deacetylation is essential for this process.
7.6. Mitochondrial Function. HDAC inhibitors, LAQ824 (dacinostat) and LBH589, induce the apoptosis of $\mathrm{E} \mu-m y c$ lymphomas via the intrinsic apoptotic pathway. Cells that inhibited the later stages of apoptosis signaling, through the knockout of apaf-1 or caspase-9, displayed morphologic features of autophagy but did not diminish the effects of LAQ824 and LBH589 on mitochondrial membrane permeabilization [39].

\section{Relationship between Autophagy and Apoptosis}

Recently, several anticancer drugs have been shown to induce not only apoptosis, but also autophagy in cancer cells [44]. The relationship between autophagy and apoptosis is complex and varies with cell types and the specific stress placed upon the cell [45]. While the molecular mechanisms leading to apoptosis have been elucidated to some extent during the past 15 years, autophagic cell death is not well characterized at the molecular level yet [46]. The induction of autophagic cell death may be an ideal approach for cancers that are resistant to apoptosis by anticancer therapies.

It is clear whether autophagy and apoptosis are strictly interconnected, as highlighted by the finding that the two 
pathways share key molecular regulators. These are summarized as follows.

(1) Caspase-3 inhibitor zDEVD-fmk upregulated autophagy in the mouse lung cancer cells [47].

(2) Following genotoxic stress or oncogene activation, the p53 tumor-suppressor protein is stabilized and activated as a transcription factor, capable of inducing apoptosis. Nuclear localization of p53 induces autophagy, whereas cytoplasmic accumulation inhibits autophagy [48].

(3) Serum- and amino acid-starved LAMP2-negative cells exhibited an accumulation of autophagic vacuoles and then succumbed to cell death with hallmarks of apoptosis, such as loss of mitochondrial transmembrane potential, caspase activation, and chromatin condensation. Together, the accumulation of autophagic vacuoles can precede apoptotic cell death [49].

(4) Beclin-1 may be a critical molecular switch that plays an important role in fine-tuning autophagy and apoptosis through caspase-9 [50].

(5) JNK and p53 may constitute an amplifying loop of autophagic and apoptotic responses. JNK activation results in phosphorylation of $\mathrm{Bcl}-2$, which enhances autophagy by disrupting the interaction between Bcl2 and Beclin-1 [51].

(6) The enforced expression of Atg5 not only promotes autophagy but also enhances susceptibility toward apoptotic stimuli irrespective of the cell type, indicating that Atg5 is a molecular switch factor between autophagy and apoptosis [52].

(7) Full-length Atg5 is an inducer of autophagy, whereas an Atg5 fragment due to cleavage by calpain is proapoptotic [53].

(8) The formation of autophagosomes occurred early in the sequence of dexamethasone-induced events, before Bak activation, loss of mitochondrial membrane potential, and nuclear fragmentation, showing that dexamethasone-induced autophagy not only lies upstream of apoptosis but is also required for the latter to occur [54].

(9) A novel Bcl-xL inhibitor, Z36, efficiently induces autophagic cell death in HeLa cells through blocking the interaction between $\mathrm{Bcl}-\mathrm{xL} / \mathrm{Bcl}-2$ and Beclin1 [55]. The antiapoptotic protein $\mathrm{Bcl}-2$ is also an antiautophagic protein through its interaction with Beclin-1 [56].

(10) The stress induced by $\mathrm{N}$-(4-hydroxyphenyl) retinamide triggers autophagy at a lower dose and apoptosis at a higher dose in glioma cells. At that time, ERK was specifically activated at a lower concentration but not at a higher concentration of retinamide [57].

(11) The kinetics of $\mathrm{Ca}^{2+}$ influx correlated well with the onset of apoptosis and autophagy, suggesting that $\mathrm{Ca}^{2+}$ might play a direct role in these processes [58].
BAPTA abolished calcium influx and significantly reduced autophagy and apoptosis.

(12) Employing 3-methyladenine, a PI3K inhibitor and specific inhibitor of the autophagy pathway, enhanced the apoptotic level [59].

These findings argue against a definitive distinction between type I (apoptotic) and type II (autophagic) cell death. The two pathways are linked in a see-saw manner, such that when apoptosis is inhibited, autophagy is initiated as a backup mechanism and vice versa $[60,61]$.

\section{Conclusions}

HDAC enzymes affect the acetylation status of histones and other important cellular proteins, which have been recognized as potentially useful therapeutic targets for a broad range of human disorders. In particular, HDAC inhibitors have emerged as major pharmacologic agents for cancer therapy. Most likely, these agents will be used in combination with standard treatment regimens. Efforts to further develop these agents should focus on the thorough evaluation of HDAC expression in different human cancers, the comprehensive analysis of the mechanisms of action of various classes of HDAC inhibitors in vitro, and the confirmation of recently identified prognosticators of response in clinical settings. However, most HDAC inhibitors target either all or at least a wide range of HDACs. This creates the problem of the unspecific inhibition of several HDACs, while the targeted blockade of specific single HDACs might be more desirable. Class-specific side effects of pan-HDAC inhibitors have been reported, supporting the requirement of selective inhibitor development. The synthesis of HDAC inhibitors that selectively target HDACs relevant to cancer initiation/progression may enhance the antitumor effects while decreasing the systemic toxicities of HDAC inhibition in cancer patients. It can be expected that the tissue- and time-specific disruption of single HDACs will uncover even more the physiological functions of particular HDACs. Although initial results examining HDAC inhibitors, administered either alone or in combination, have been disappointing, attention has now turned to more potent and selective compounds that may prove to be more effective.

\section{Abbreviations}

AIF: Apoptosis-inducing factor

APAF-1: Apoptotic protease-activating factor 1

Atg: Autophagy-related gene

BAPTA: 1,2-bis(o-aminophenoxy)ethane-N,N, $\mathrm{N}^{\prime}, \mathrm{N}^{\prime}$ tetraacetic acid

Beclin-1: Bcl-2-interacting protein

CDKs: Cyclin-dependent kinases

ERK: Extracellular signal-regulated kinase

FADD: Fas-associated death domain

HDAC: Histone deacetylase

Htr-A2: High temperature requirement factor A2

JNK1: c-Jun N-terminal kinase 1

LAMP2: Lysosome-associated membrane glycoprotein 2 
LC3: Microtubule-associated protein 1 light chain 3

mTOR: Mammalian target of rapamycin

PCD: Programmed cell death

PI3K: Phosphatidylinositol 3-kinase

ROS: Reactive oxygen species

SAHA: Suberoylanilide hydroxamic acid

siRNA: Small interfering RNA

SMAC: Second mitochondria-derived activator of caspase

S6rp: S6 protein of the 40S ribosomal subunit

TRAIL: TNF-related apoptosis-inducing ligand

TSA: Trichostatin-A

XIAP: X-linked inhibitor of apoptosis protein.

\section{Acknowledgments}

The author apologizes to all colleagues whose work may not have been cited for space reasons. The author is grateful to D. Mrozek for editing the manuscript. The authors' work described in this paper was supported in part by a Grantin-Aid for Scientific Research (20659309) from the Japan Society for the Promotion of Science.

\section{References}

[1] Y. Shao, Z. Gao, P. A. Marks, and X. Jiang, "Apoptotic and autophagic cell death induced by histone deacetylase inhibitors," Proceedings of the National Academy of Sciences of the United States of America, vol. 101, no. 52, pp. 18030-18035, 2004.

[2] M. Esteller, "Molecular origins of cancer: epigenetics in cancer," New England Journal of Medicine, vol. 358, no. 11, pp. 1148-1159, 2008.

[3] A. L. Clayton, C. A. Hazzalin, and L. C. Mahadevan, "Enhanced histone acetylation and transcription: a dynamic perspective," Molecular Cell, vol. 23, no. 3, pp. 289-296, 2006.

[4] B. D. Strahl and C. D. Allis, "The language of covalent histone modifications," Nature, vol. 403, no. 6765, pp. 41-45, 2000.

[5] S. Balasubramanian, E. Verner, and J. J. Buggy, "Isoformspecific histone deacetylase inhibitors: the next step?" Cancer Letters, vol. 280, no. 2, pp. 211-221, 2009.

[6] E. A. Olsen, Y. H. Kim, T. M. Kuzel et al., "Phase IIb multicenter trial of vorinostat in patients with persistent, progressive, or treatment refractory cutaneous T-cell lymphoma," Journal of Clinical Oncology, vol. 25, no. 21, pp. 3109-3115, 2007.

[7] H. J. Mackay, H. Hirte, T. Colgan et al., "Phase II trial of the histone deacetylase inhibitor belinostat in women with platinum resistant epithelial ovarian cancer and micropapillary (LMP) ovarian tumours," European Journal of Cancer, vol. 46, no. 9, pp. 1573-1579, 2010.

[8] D. M. Vigushin, S. Ali, P. E. Pace et al., "Trichostatin A is a histone deacetylase inhibitor with potent antitumor activity against breast cancer in vivo," Clinical Cancer Research, vol. 7, no. 4, pp. 971-976, 2001.

[9] J. S. De Bono, R. Kristeleit, A. Tolcher et al., "Phase I pharmacokinetic and pharmacodynamic study of LAQ824, a hydroxamate histone deacetylase inhibitor with a heat shock protein-90 inhibitory profile, in patients with advanced solid tumors," Clinical Cancer Research, vol. 14, no. 20, pp. 6663 6673, 2008.
[10] G. Lopez, J. Liu, W. Ren et al., "Combining PCI-24781, a novel histone deacetylase inhibitor, with chemotherapy for the treatment of soft tissue sarcoma," Clinical Cancer Research, vol. 15, no. 10, pp. 3472-3483, 2009.

[11] M. Dickinson, D. Ritchie, D. J. DeAngelo et al., "Preliminary evidence of disease response to the pan deacetylase inhibitor panobinostat (LBH589) in refractory Hodgkin Lymphoma," British Journal of Haematology, vol. 147, no. 1, pp. 97-101, 2009.

[12] C. Grant, F. Rahman, R. Piekarz et al., "Romidepsin: a new therapy for cutaneous T-cell lymphoma and a potential therapy for solid tumors," Expert Review of Anticancer Therapy, vol. 10, no. 7, pp. 997-1008, 2010.

[13] K. B. Glaser, "HDAC inhibitors: clinical update and mechanism-based potential," Biochemical Pharmacology, vol. 74, no. 5, pp. 659-671, 2007.

[14] A. Hauschild, U. Trefzer, C. Garbe et al., "Multicenter phase II trial of the histone deacetylase inhibitor pyridylmethyl-N$\{4$-[(2-aminophenyl)-carbamoyl]-benzyl $\}$-carbamate in pretreated metastatic melanoma," Melanoma Research, vol. 18, no. 4, pp. 274-278, 2008.

[15] P. Munster, D. Marchion, E. Bicaku et al., "Clinical and biological effects of valproic acid as a histone deacetylase inhibitor on tumor and surrogate tissues: phase I/II trial of valproic acid and epirubicin/FEC," Clinical Cancer Research, vol. 15, no. 7, pp. 2488-2496, 2009.

[16] L. McMahon, H. Tamary, M. Askin et al., "A randomized phase II trial of Arginine Butyrate with standard local therapy in refractory sickle cell leg ulcers," British Journal of Haematology, vol. 151, no. 5, pp. 516-524, 2010.

[17] W. S. Xu, R. B. Parmigiani, and P. A. Marks, "Histone deacetylase inhibitors: molecular mechanisms of action," Oncogene, vol. 26, no. 37, pp. 5541-5552, 2007.

[18] P. A. Marks and W.-S. Xu, "Histone deacetylase inhibitors: potential in cancer therapy," Journal of Cellular Biochemistry, vol. 107, no. 4, pp. 600-608, 2009.

[19] W. Weichert, A. Roske, V. Gekeler et al., "Histone deacetylases 1,2 and 3 are highly expressed in prostate cancer and HDAC2 expression is associated with shorter PSA relapse time after radical prostatectomy," British Journal of Cancer, vol. 98, no. 3, pp. 604-610, 2008.

[20] L. Galluzzi, M. C. Maiuri, I. Vitale et al., "Cell death modalities: classification and pathophysiological implications," Cell Death and Differentiation, vol. 14, no. 7, pp. 1237-1243, 2007.

[21] G. Kroemer and M. Jaattela, "Lysosomes and autophagy in cell death control," Nature Reviews Cancer, vol. 5, no. 11, pp. 886$897,2005$.

[22] R. R. Rosato, J. A. Almenara, Y. Dai, and S. Grant, "Simultaneous activation of the intrinsic and extrinsic pathways by histone deacetylase (HDAC) inhibitors and tumor necrosis factor-related apoptosis-inducing ligand (TRAIL) synergistically induces mitochondrial damage and apoptosis in human leukemia cells," Molecular Cancer Therapeutics, vol. 2, no. 12, pp. 1273-1284, 2003.

[23] J. Xu, J.-Y. Zhou, W.-Z. Wei, S. Philipsen, and G. S. Wu, "Sp1mediated TRAIL induction in chemosensitization," Cancer Research, vol. 68, no. 16, pp. 6718-6726, 2008.

[24] Y. Dai, M. Rahmani, P. Dent, and S. Grant, "Blockade of histone deacetylase inhibitor-induced RelA/p65 acetylation and NF- $\kappa \mathrm{B}$ activation potentiates apoptosis in leukemia cells through a process mediated by oxidative damage, XIAP downregulation, and c-Jun N-terminal kinase 1 activation," Molecular and Cellular Biology, vol. 25, no. 13, pp. 5429-5444, 2005. 
[25] S. Fulda, "Modulation of TRAIL-induced apoptosis by HDAC inhibitors," Current Cancer Drug Targets, vol. 8, no. 2, pp. 132140, 2008.

[26] M. Donadelli, C. Costanzo, S. Beghelli et al., "Synergistic inhibition of pancreatic adenocarcinoma cell growth by trichostatin A and gemcitabine," Biochimica et Biophysica Acta, vol. 1773, no. 7, pp. 1095-1106, 2007.

[27] P. Garcia-Morales, A. Gomez-Martinez, A. Carrato et al., "Histone deacetylase inhibitors induced caspase-independent apoptosis in human pancreatic adenocarcinoma cell lines," Molecular Cancer Therapeutics, vol. 4, no. 8, pp. 1222-1230, 2005.

[28] H. Y. Cohen, C. Miller, K. J. Bitterman et al., "Calorie restriction promotes mammalian cell survival by inducing the SIRT1 deacetylase," Science, vol. 305, no. 5682, pp. 390-392, 2004.

[29] P. Fritsche, B. Seidler, S. Schüler et al., "HDAC2 mediates therapeutic resistance of pancreatic cancer cells via the $\mathrm{BH} 3-$ only protein NOXA," Gut, vol. 58, no. 10, pp. 1399-1409, 2009.

[30] J. S. Carew, S. T. Nawrocki, and J. L. Cleveland, "Modulating autophagy for therapeutic benefit," Autophagy, vol. 3, no. 5, pp. 464-467, 2007.

[31] J. S. Carew, E. C. Medina, J. A. Esquivel et al., "Autophagy inhibition enhances vorinostat-induced apoptosis via ubiquitinated protein accumulation," Journal of Cellular and Molecular Medicine, vol. 14, no. 10, pp. 2448-2459, 2010.

[32] S. Yamamoto, K. Tanaka, R. Sakimura et al., "Suberoylanilide hydroxamic acid (SAHA) induces apoptosis or autophagyassociated cell death in chondrosarcoma cell lines," Anticancer Research, vol. 28, no. 3A, pp. 1585-1591, 2008.

[33] A. Hrzenjak, M.-L. Kremser, B. Strohmeier et al., "SAHA induces caspase-independent, autophagic cell death of endometrial stromal sarcoma cells by influencing the mTOR pathway," Journal of Pathology, vol. 216, no. 4, pp. 495-504, 2008.

[34] M. Watanabe, S. Adachi, H. Matsubara et al., "Induction of autophagy in malignant rhabdoid tumor cells by the histone deacetylase inhibitor FK228 through AIF translocation," International Journal of Cancer, vol. 124, no. 1, pp. 55-67, 2009.

[35] J. S. Carew, S. T. Nawrocki, C. N. Kahue et al., "Targeting autophagy augments the anticancer activity of the histone deacetylase inhibitor SAHA to overcome Bcr-Abl-mediated drug resistance," Blood, vol. 110, no. 1, pp. 313-322, 2007.

[36] J. Long, J. Zhao, Z. Yan, Z. Liu, and N. Wang, "Antitumor effects of a novel sulfur-containing hydroxamate histone deacetylase inhibitor H40," International Journal of Cancer, vol. 124, no. 5, pp. 1235-1244, 2009.

[37] M. Oh, I.-K. Choi, and H. J. Kwon, "Inhibition of histone deacetylase1 induces autophagy," Biochemical and Biophysical Research Communications, vol. 369, no. 4, pp. 1179-1183, 2008.

[38] J.-Y. Lee, H. Koga, Y. Kawaguchi et al., "HDAC6 controls autophagosome maturation essential for ubiquitin-selective quality-control autophagy," EMBO Journal, vol. 29, no. 5, pp. 969-980, 2010.

[39] L. Ellis, M. Bots, R. K. Lindemann et al., "The histone deacetylase inhibitors LAQ824 and LBH589 do not require death receptor signaling or a functional apoptosome to mediate tumor cell death or therapeutic efficacy," Blood, vol. 114, no. 2, pp. 380-393, 2009.

[40] Y.-P. Yang, Z.-Q. Liang, Z.-L. Gu, and Z.-H. Qin, "Molecular mechanism and regulation of autophagy," Acta Pharmacologica Sinica, vol. 26, no. 12, pp. 1421-1434, 2005.
[41] C. Yu, M. Subler, M. Rahmani et al., "Induction of apoptosis in BCR/ABL+ cells by histone deacetylase inhibitors involves reciprocal effects on the RAF/MEK/ERK and JNK pathways," Cancer Biology \& Therapy, vol. 2, no. 5, pp. 544-551, 2003.

[42] A. J. Wilson, D.-S. Byun, N. Popova et al., "Histone deacetylase 3 (HDAC3) and other class I HDACs regulate colon cell maturation and p21 expression and are deregulated in human colon cancer," Journal of Biological Chemistry, vol. 281, no. 19, pp. 13548-13558, 2006.

[43] D. Mottet, S. Pirotte, V. Lamour et al., "HDAC4 represses p21 (WAF1/Cip1) expression in human cancer cells through a Sp1dependent, p53-independent mechanism," Oncogene, vol. 28, no. 2, pp. 243-256, 2009.

[44] K. S. Song, J. S. Kim, E. J. Yun et al., "Rottlerin induces autophagy and apoptotic cell death through a PKC-deltaindependent pathway in HT1080 human fibrosarcoma cells: the protective role of autophagy in apoptosis," Autophagy, vol. 4, no. 5, pp. 650-658, 2008.

[45] H. Zhang, X. Kong, J. Kang et al., "Oxidative stress induces parallel autophagy and mitochondria dysfunction in human glioma U251 cells," Toxicological Sciences, vol. 110, no. 2, pp. 376-388, 2009.

[46] N. Kourtis and N. Tavernarakis, "Autophagy and cell death in model organisms," Cell Death and Differentiation, vol. 16, no. 1, pp. 21-30, 2009.

[47] K. W. Kim, M. Hwang, L. Moretti, J. J. Jaboin, Y. I. Cha, and B. Lu, "Autophagy upregulation by inhibitors of caspase-3 and $\mathrm{mTOR}$ enhances radiotherapy in a mouse model of lung cancer," Autophagy, vol. 4, no. 5, pp. 659-668, 2008.

[48] E. Morselli, E. Tasdemir, M. C. Maiuri et al., "Mutant p53 protein localized in the cytoplasm inhibits autophagy," Cell Cycle, vol. 7, no. 19, pp. 3056-3061, 2008.

[49] R.-A. Gonzalez-Polo, P. Boya, A.-L. Pauleau et al., “The apoptosis/autophagy paradox: autophagic vacuolization before apoptotic death," Journal of Cell Science, vol. 118, no. 14, pp. 3091-3102, 2005.

[50] Z.-H. Wang, L. Xu, Z.-L. Duan et al., "Beclin 1-mediated macroautophagy involves regulation of caspase- 9 expression in cervical cancer HeLa cells," Gynecologic Oncology, vol. 107, no. 1, pp. 107-113, 2007.

[51] S. Lorin, A. Borges, L. R. Dos Santos et al., "c-Jun NH2terminal kinase activation is essential for DRAM-dependent induction of autophagyand apoptosis in 2-methoxyestradioltreated Ewing sarcoma cells," Cancer Research, vol. 69, no. 17, pp. 6924-6931, 2009.

[52] A. Bommareddy, E. R. Hahm, D. Xiao et al., "Atg5 regulates phenethyl isothiocyanate-induced autophagic and apoptotic cell death in human prostate cancer cells," Cancer Research, vol. 69, no. 8, pp. 3704-3712, 2009.

[53] S. Yousefi, R. Perozzo, I. Schmid et al., "Calpain-mediated cleavage of Atg5 switches autophagy to apoptosis," Nature Cell Biology, vol. 8, no. 10, pp. 1124-1132, 2006.

[54] E. Laane, K. P. Tamm, E. Buentke et al., "Cell death induced by dexamethasone in lymphoid leukemia is mediated through initiation of autophagy," Cell Death and Differentiation, vol. 16, no. 7, pp. 1018-1029, 2009.

[55] J. Lin, Z. Zheng, Y. Li et al., "A novel Bcl-XL inhibitor Z36 that induces autophagic cell death in HeLa cells," Autophagy, vol. 5, no. 3, pp. 314-320, 2009.

[56] S. Pattingre, A. Tassa, X. Qu et al., "Bcl-2 antiapoptotic proteins inhibit Beclin 1-dependent autophagy," Cell, vol. 122, no. 6, pp. 927-939, 2005. 
[57] M. Tiwari, V. K. Bajpai, A. A. Sahasrabuddhe et al., "Inhibition of $\mathrm{N}$-(4-hydroxyphenyl)retinamide-induced autophagy at a lower dose enhances cell death in malignant glioma cells," Carcinogenesis, vol. 29, no. 3, pp. 600-609, 2008.

[58] M. Shi, H.-N. Wang, S.-T. Xie et al., "Antimicrobial peptaibols, novel suppressors of tumor cells, targeted calcium-mediated apoptosis and autophagy in human hepatocellular carcinoma cells," Molecular Cancer, vol. 9, no. 1, pp. 26-40, 2010.

[59] Q. Cui, S.-I. Tashiro, S. Onodera, and T. Ikejima, "Augmentation of oridonin-induced apoptosis observed with reduced autophagy," Journal of Pharmacological Sciences, vol. 101, no. 3, pp. 230-239, 2006.

[60] P. Boya, R.-A. González-Polo, N. Casares et al., "Inhibition of macroautophagy triggers apoptosis," Molecular and Cellular Biology, vol. 25, no. 3, pp. 1025-1040, 2005.

[61] L. Ellis and R. Pili, "Histone deacetylase inhibitors: advancing therapeutic strategies in hematological and solid malignancies," Pharmaceuticals, vol. 3, no. 8, pp. 2411-2469, 2010. 

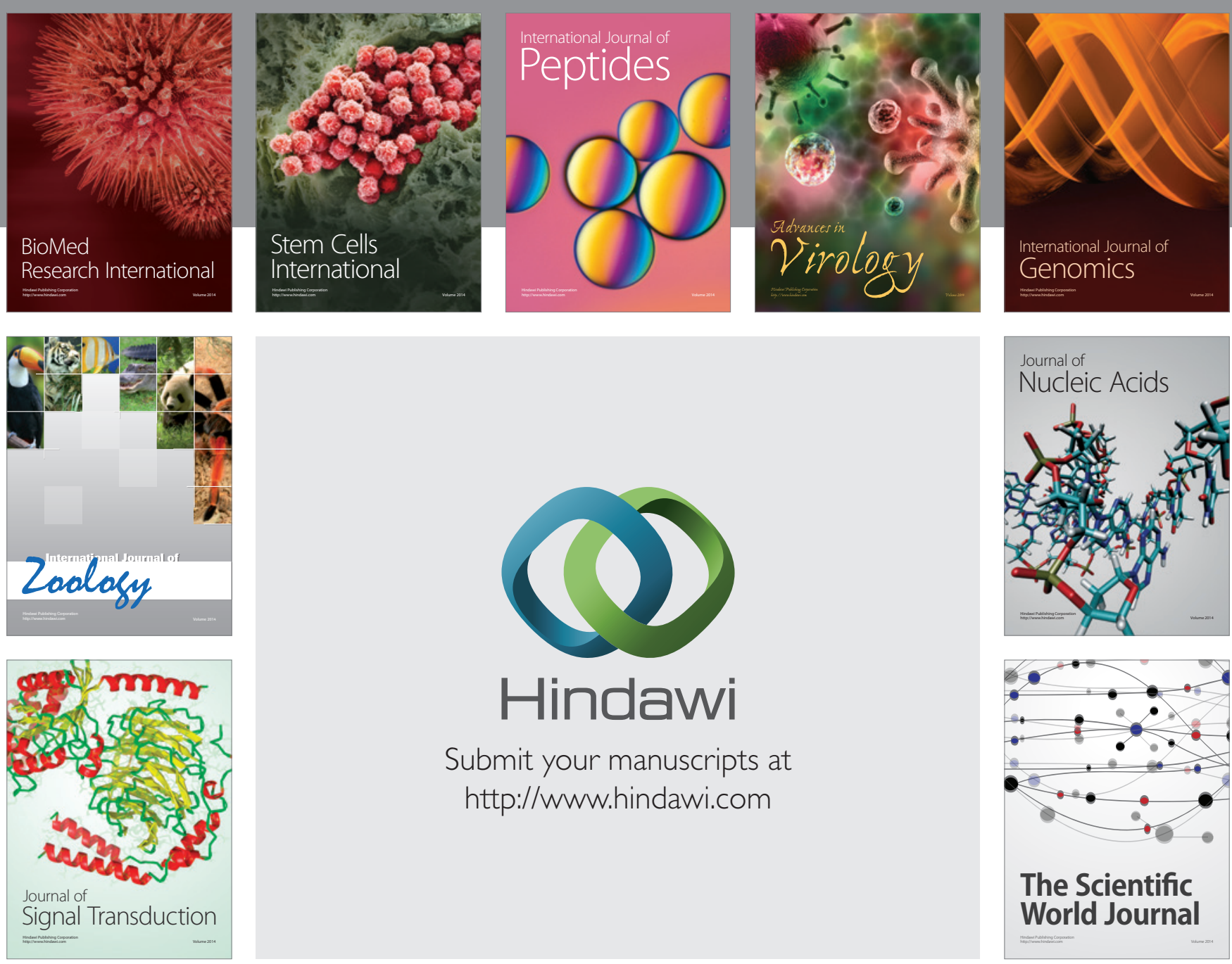

Submit your manuscripts at

http://www.hindawi.com
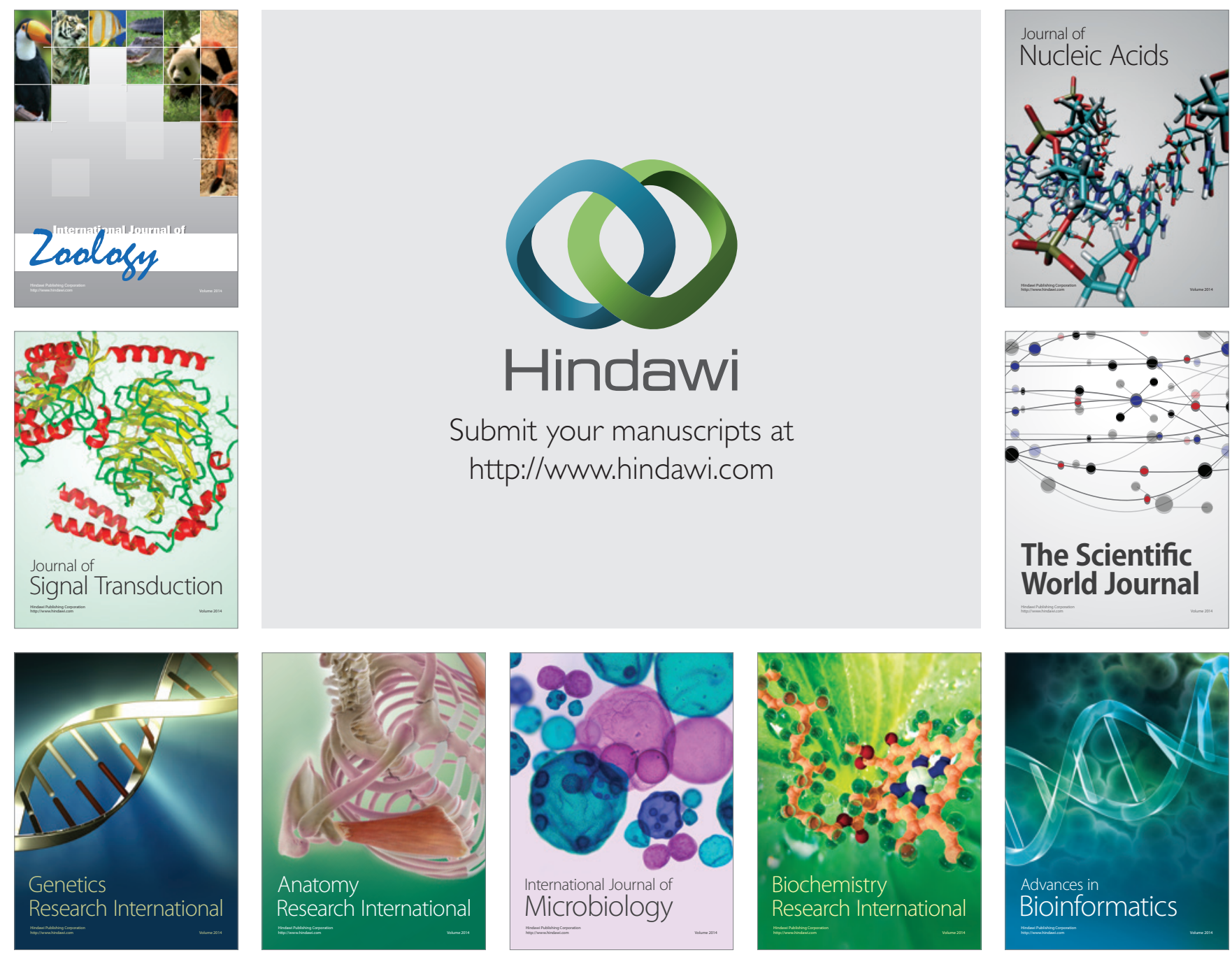

The Scientific World Journal
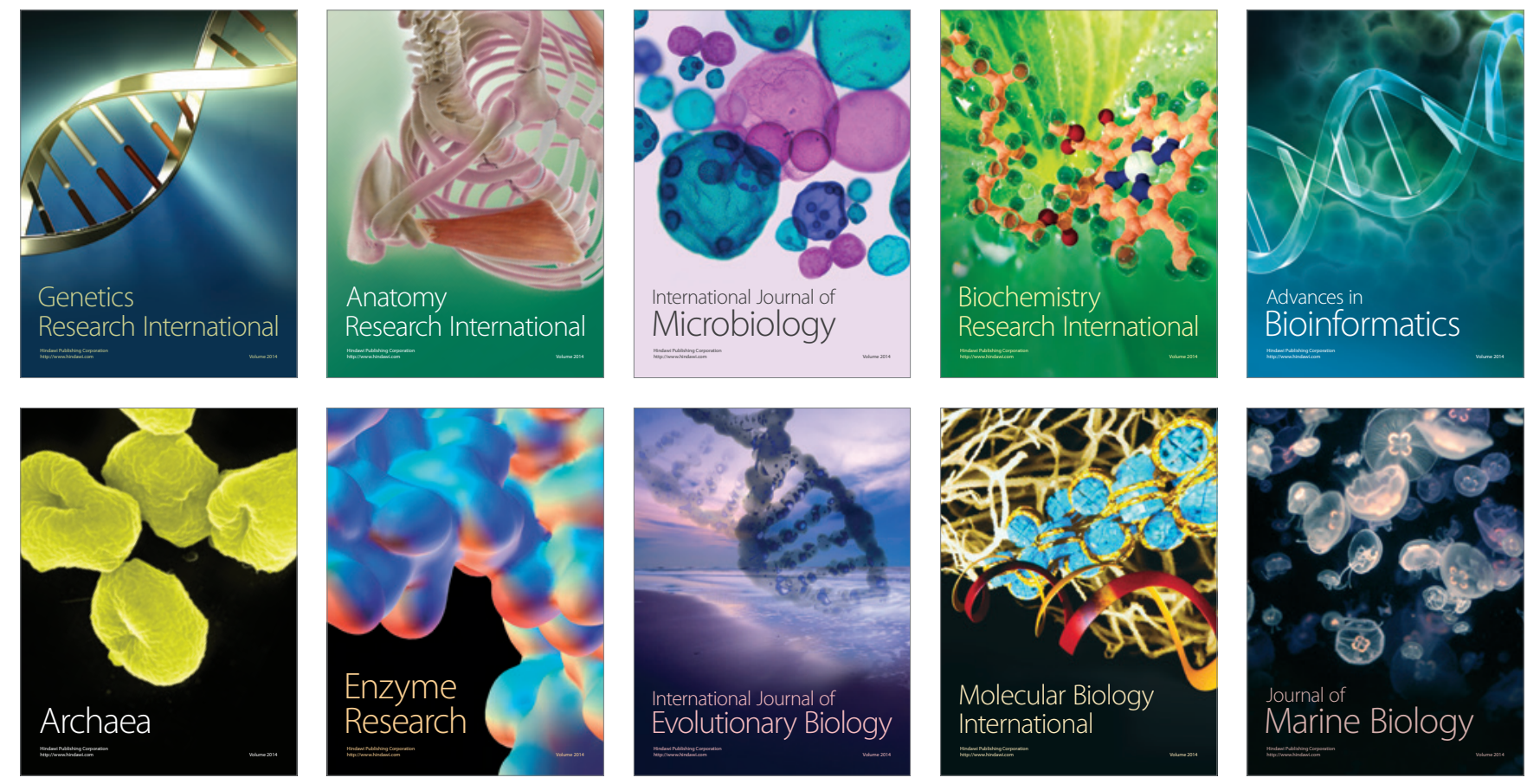